\title{
Repercussões da pandemia da covid-19 nos profissionais de saúde: como está quem está na linha de frente?
}

\section{Covid-19 pandemic repercussions on healthcare professionals: how is who on the front line?}

DOI: $10.46919 / \operatorname{archv} 2 n 3-014$

Recebimento dos originais: 01/01/2021

Aceitação para publicação: 31/03/2021

\author{
Andressa Karine Schons \\ Acadêmica do curso de Bacharelado em Enfermagem \\ Instituição: Fundação Educacional Machado de Assis-FEMA \\ Endereço: R. Santos Dumont, 820 - Centro, Santa Rosa - RS \\ E-mail: andressaschons@ outlook.com
}

\section{Andressa Fátima May}

Acadêmica do curso de Bacharelado em Enfermagem Instituição: Universidade Luterana do Brasil, Carazinho - ULBRA

Endereço: R. Três de Outubro, 378 - Vargas, Carazinho - RS

E-mail: andressamaypl@hotmail.com

\section{Claudia Teresinha Muller}

Acadêmica do curso de Bacharelado em Enfermagem Instituição: Fundação Educacional Machado de Assis- FEMA

Endereço: R. Santos Dumont, 820 - Centro, Santa Rosa - RS

E-mail: claudiamuller261@gmail.com

Flávia Michelle Pereira Albuquerque

Mestre em Desenvolvimento e Políticas Públicas

Instituição: Universidade Federal da Fronteira Sul - UFFS

Endereço: av Francisco timm 480 - santa rosa - RS

E-mail: flaviampa@msn.com

Edenilson Freitas Rodrigues

Mestre em Desenvolvimento e Políticas Públicas

Instituição: Universidade Federal da Fronteira Sul - UFFS

Endereço: av Francisco timm 480 - santa rosa - RS

E-mail: edenilsonfrodrigues@gmail.com

\section{Michele Silva Lachno}

Especialista em saúde da família

Instituição: Universidade Regional do Noroeste do Estado do Rio Grande do Sul - Unijui

Endereço: av Francisco timm 480 - santa rosa - RS

E-mail: milachno@yahoo.com.br 


\title{
RESUMO
}

No cenário da pandemia da COVID-19, emerge enquanto protagonistas no cuidado coletivo, os trabalhadores de saúde que estão prestando à assistência direta e indireta a população. Este estudo tinha como objetivo verificar as repercussões da pandemia da COVID-19 na saúde mental, do trabalho e na física, dos profissionais de saúde da Fundação Municipal de Saúde de Santa Rosa (FUMSSAR). O estudo foi desenvolvido nas unidades de saúde da FUMSSAR. A coleta de dados foi realizada através de questionário autoaplicável, durante os meses de julho e setembro de 2020. Participaram do estudo 140 trabalhadores. Quando questionados sobre diagnósticos pré-existentes de doenças crônicas não transmissíveis (DCNT), a maioria $80(70,4 \%)$ afirmou não possuir nenhum diagnóstico prévio de DCNT. Em relação à COVID-19, a maioria - $71(56,8 \%)$ - dos entrevistados afirmam não se sentirem seguros, em relação ao contágio pelo novo coronavírus, em seus locais de trabalho. Apesar do medo referido e do sentimento de insegurança na atuação, $61(48,8 \%)$ não se afastaria do trabalho. Os resultados obtidos neste estudo auxiliam no delineamento da dimensão ocupacional durante a pandemia. Fatores como os sentimentos de medo quando da possibilidade de contaminação de familiares, transparece os elementos que constituem o sujeito para além do trabalho exercido, humanizando tais cuidadores. Espera-se que esta pesquisa auxilie na tomada de decisões em saúde voltadas para os profissionais de saúde que estão atuando na linha de frente no enfrentamento a pandemia da COVI-19.

Palavras-chave: Coronavírus, Sentimentos, Profissionais da Saúde.

\begin{abstract}
In the COVID-19 pandemic scenario, health workers who are providing direct and indirect assistance to the population emerge as protagonists in collective care. This study aimed to verify the the repercussions of the COVID-19 pandemic on the mental, work and physical health of health professionals at the Municipal Health Foundation of Santa Rosa (FUMSSAR). The study was developed in the health units of FUMSSAR. Data collection was performed through a self-administered questionnaire during the months of July and September 2020. 140 workers participated in the study. When asked about pre-existing diagnoses of chronic non-communicable diseases (NCDs), the majority $80(70.4 \%)$ stated that they had no previous diagnosis of NCDs. In relation to COVID-19, the majority - 71 (56.8\%) - of the interviewees affirm that they do not feel safe, in relation to infection by the new coronavirus, in their workplaces. Despite the referred fear and the feeling of insecurity in the performance, 61 (48.8\%) would not leave work. The results obtained in this study help to outline the occupational dimension during the pandemic.Factors such as feelings of fear when there is the possibility of contamination by family members, the elements that constitute the subject, in addition to the work carried out, become humanized. It is hoped that this research will assist in making health decisions aimed at health professionals who are working on the front line in coping with the COVI19 pandemic.
\end{abstract}

Keywords: Pandemic, Feelings, Health Professionals.

\section{INTRODUÇÃO}

O Brasil e o mundo têm atualmente a necessidade de enfrentamento aos efeitos ocorridos da COVID-19. Diversos aspectos da vida social sofreram impactos em um curto período de tempo, onde a Organização Mundial de Saúde (OMS) em março de 2020 declara pandemia, fazendo com que nações atentem para a necessidade de estratégia de minimizar os danos à saúde da coletividade. 
A OMS no último dia do ano de 2019 monitorou casos de pneumonia na cidade de Wuhan na China, possivelmente relacionados a um novo tipo de coronavírus até então desconhecidos em seres humanos. Na primeira semana de 2020, o novo vírus é identificado pelas autoridades chinesas.

Em 11 de fevereiro de 2020 o novo coronavírus recebe a denominação de SARS COV-2, sendo responsável por causar a doença COVID-19. A OMS e a OPAS trabalham em conjunto para auxiliar os países a entenderem o comportamento do vírus, como se dá a sua transmissibilidade, tratamentos e como os países tem conseguido lidar com essa pandemia.

A COVID-19 é uma doença causada pelo coronavírus SARS-CoV-2, que apresenta um quadro clínico que varia de infecções assintomáticas a quadros respiratórios graves. De acordo com a Organização Mundial de Saúde (OMS), a maioria dos pacientes com COVID-19 (cerca de 80\%) podem ser assintomáticos e cerca de $20 \%$ dos casos podem requerer atendimento hospitalar por apresentarem dificuldade respiratória e desses casos aproximadamente $5 \%$ podem necessitar de suporte para o tratamento de insuficiência respiratória (suporte ventilatório).

Coronavírus é uma família de vírus que causam infecções respiratórias. O novo agente do coronavírus foi descoberto em 31 de dezembro de 2019 após casos registrados na China. Provoca a doença chamada de coronavírus (COVID-19).

Os primeiros coronavírus humanos foram isolados pela primeira vez em 1937. No entanto, foi em 1965 que o vírus foi descrito como coronavírus, em decorrência do perfil na microscopia, parecendo uma coroa.

A maioria das pessoas se infecta com os coronavírus comuns ao longo da vida, sendo as crianças pequenas mais propensas a se infectar com o tipo mais comum do vírus. Os coronavírus mais comuns que infectam humanos são o alpha coronavírus 229E e NL63 e beta coronavírus OC43, HKU1.

Os sintomas da COVID-19 podem variar de um simples resfriado até uma pneumonia severa. Sendo os sintomas mais comuns: Tosse, Febre, Coriza, Dor de garganta e Dificuldade para respirar A transmissão acontece de uma pessoa doente para outra ou por contato próximo por meio de: Toque do aperto de mão; Gotículas de saliva; Espirro; Tosse; Catarro; Objetos ou superfícies contaminadas, como celulares, mesas, maçanetas, brinquedos, teclados de computador etc.

Neste cenário, emerge enquanto protagonistas no cuidado coletivo, os trabalhadores de saúde que estão na linha de frente prestando a assistência de maneira direta e indireta à população. Acrescido a isso cabe lembrança que o trabalhador da área da saúde tem estado exposto a um cenário amplamente dinâmico quanto orientações técnicas, garantia de insumos, equipamentos e incertezas a êxitos das condutas terapêuticas.

Os profissionais de saúde envolvidos nesse senário estão expostos diariamente ao risco de adoecer pelo coronavírus, sendo que a diferença que caracteriza este contingente da força de trabalho determina 
formas diferentes de exposição, tanto ao risco de contaminação quanto aos fatores associados às condições de trabalho (SOUZA E SOUZA e SOUZA, 2020). Problemas como cansaço físico e estresse psicológico, insuficiência e/ou negligência com relação às medidas de proteção e cuidado à saúde desses profissionais.

Trabalhar em meio a uma pandemia exige dos profissionais e dos serviços uma estrutura consistente capaz de comandar e controlar a tomada de decisões e as informações, permitindo que estes possam atravessar a pandemia da melhor forma. Em Santa Rosa, município do noroeste do Rio Grande do Sul, não diferente do restante do país, há uma organização na prestação da assistência em saúde voltada à sua peculiar realidade. A Fundação Municipal de Saúde de Santa Rosa -FUMSSAR- conta com o serviço de referência em saúde do trabalhador, CEREST Fronteira Noroeste, que assiste tecnicamente a 22 municípios da Região 14 do Rio Grande do Sul.

Com isso propõe-se um estudo que visa realizar um levantamento dos principais aspectos na vida e na saúde dos profissionais de saúde que estão na linha de frente no combate a pandemia da COVID-19, dessa maneira facilitará o desenvolvimento de atividades/treinamentos e educação continuada de modo a fornecer subsídios necessários para qualificar não só a assistência prestada como também consolidar e assegurar os profissionais diante deste senário vivenciado mundialmente.

\section{METODOLOGIA}

\subsection{TIPO DE ESTUDO:}

A caracterização desse estudo configura-se como uma pesquisa de abordagem tipo quantitativa, do tipo descritivo e explicativo, com delineamento transversal.

Sampieri, Colado e Baptista (2013), define a pesquisa quantitativa como a metodologia mais objetiva possível, onde se utiliza à lógica ou o raciocínio dedutivo para generalizar os resultados encontrados em um grupo maior, além disso, este tipo de abordagem permite a possibilidade de réplica do estudo ou enfoque sobre pontos específicos dos fenômenos, permitindo a comparação entre estudos similares. Além disso, para os mesmos autores, os estudos descritivos buscam identificar as propriedades e características de determinados grupos, processos ou fenômenos a fim de revelar com exatidão as dimensões de um fenômeno ou contexto.

O delineamento do estudo é transversal, pois os dados foram coletados em um só momento projetando um registro sobre tal situação (SAMPIERI; COLLADO; BAPTISTA, 2013).

\subsection{UNIDADE DE ANÁLISE:}

Este estudo será realizado no município de Santa Rosa/RS, que possui um quantitativo de 72.919 habitantes (IBGE, 2020), sendo um polo regional na área da saúde nos diferentes níveis de atenção, 
contemplando desde ações preventivas e diferenciais na $\mathrm{AB}$ até serviços e ações na área da alta complexidade (FUMSSAR, 2016).

O estudo foi desenvolvido nas unidades de saúde da FUMSSAR, que possui 434 (quatrocentos e trinta e quatro) funcionários trabalhando na linha de frente de combate a pandemia. Atualmente a FUMSSAR conta com 18 (dezoito) Unidades Básicas de Saúde e três unidades sentinelas, além de, dois CAPS, um CER II e um CEREST. Foram sujeitos do estudo, trabalhadores da área da saúde, na atenção básica, que estavam atuando na linha de frente da pandemia de COVID-19, no momento da coleta de dados, que estivessem atuando em estabelecimentos de saúde credenciados junto ao Cadastro Nacional de Estabelecimentos de Saúde (CNES).

Responderam ao questionário 140 trabalhadores da área da saúde que estão atuando na linha de frente da pandemia de COVID-19 do setor público de saúde do município em unidades básicas de saúde e unidades sentinela.

\subsection{SUJEITOS DA PESQUISA:}

O estudo realizou-se com cento e quarenta profissionais da área da saúde do município de Santa Rosa/RS, que estão atuando em estabelecimentos de saúde credenciados junto ao Cadastro Nacional de Estabelecimentos de Saúde (CNES) e que estão atuando na linha de frente da COVID-19 no ano de 2020.

\subsection{COLETA DE DADOS:}

O instrumento de coleta de dados foi submetido a um pré-teste com participantes semelhante ao grupo estudado, participando três enfermeiros, 01 técnico de enfermagem, 01 fonoaudióloga e 01 médica. Estes foram escolhidos no município de Santa Rosa, com equipes de saúde semelhantes. Com o pré-teste do instrumento foi possível avaliar a compreensão das questões, assim como realizar as alterações necessárias previamente à etapa de coleta de dados.

A coleta de dados efetivou-se através do google forms, contendo trinta e cinco questões de múltipla escolha no período de julho a setembro de 2020. O questionário contem questões acerca do perfil Sociodemográfico dos participantes, descrição da atuação profissional e aspectos relacionados ao COVID19.

\subsection{ANÁLISE E INTERPRETAÇÃO DOS DADOS:}

As variáveis categóricas serão apresentadas em percentuais. Para avaliar as diferenças nas variáveis categóricas. Foi utilizado o teste de qui-quadrado. Para todas as análises, foi considerado um nível de significância de 5\%. A análise estatística foi realizada com auxílio só software SPSS versão 22.0. 


\subsection{PRINCÍPIOS ÉTICOS NA PESQUISA:}

A pesquisa respeitou a Resolução CNS nº66/2012 do Conselho Nacional de Saúde do Ministério da Saúde, como também o Código de Ética Profissional dos Psicólogos. Considerar-se-á os seguintes aspectos éticos: Aprovação pelo Comitê Científico do Núcleo de Ensino e Pesquisa (NEP) da Fundação Municipal de Saúde de Santa Rosa. Parecer Consubstanciado do Comitê de Ética em Pesquisa (CEP) aprovado sob o n ${ }^{\circ}$ 4.233.095 e CAAE 33172920.6.0000.5331. Termo de Consentimento Livre e Esclarecido (TCLE).

\section{RESULTADOS}

Nesta pesquisa responderam ao questionário 140 profissionais da área da saúde, que estão na linha de frente, com idade entre 18 a 60 anos ou mais, sendo que 29,28\% tinham entre 18 e 29 anos, desvelando equipe muito jovem. Quanto aos gêneros das equipes 08,57\% pessoas são do sexo masculino e 91,42\% pessoas do sexo feminino. De modo similar o gênero associa-se à elaboração cultural, então os gêneros masculino e feminino são construções culturais, sendo capaz de ter oscilações no tempo e no espaço que estão em constantes formações, por conseguinte o sexo relaciona-se ao biológico (RAVAGANI, 2007).

Ao considerar a finalidade de compreender as reações e os sentimentos de profissionais da linha de frente, no atendimento a pacientes com suspeita e/ou confirmados de COVID-19, foram entrevistados os profissionais que trabalham na FUMMSAR, setor público municipal. Desta amostra 37,14\% declaram estado civil solteiro (a) e 36,42\% casados tendo em vista que grande parte das pessoas participantes tem ensino médio e técnico (47,14\%), do mesmo modo que possuem graduados $(18,57 \%)$ e especialistas $(29,28 \%)$ na área de atuação. Já em relação ao tempo de trabalho dos profissionais entrevistados na instituição do estudo, $10,71 \%$ referiram trabalhar entre zero a seis meses; $13,57 \%$ entre dose meses a vinte e quatro meses; mais de quarenta e oito meses cerca de $60,71 \%$.

Tabela 1 - Perfil Sociodemográfico

\begin{tabular}{l|l|}
\hline Variáveis & $\begin{array}{l}\text { Percentual dos } \\
\text { usuários (N=140) } \\
\mathrm{n} \%\end{array}$ \\
\hline Sexo & $08,57 \%$ \\
Masculino & $91,42 \%$ \\
Feminino & \\
\hline Grau de instrução & $47,14 \%$ \\
Ensino Médio e Técnico & $18,57 \%$ \\
Ensino Superior & $29,28 \%$ \\
Especialização & $04,28 \%$ \\
Mestrado & $00,71 \%$ \\
Doutorado & \\
\hline Estado Civil & $36,42 \%$ \\
Casado & $37,14 \%$ \\
Solteiro & $17,14 \%$ \\
União estável & \\
\hline
\end{tabular}




\begin{tabular}{l|l}
\hline Separado ou divorciado & $06,42 \%$ \\
Viúvo (a) & $02,85 \%$ \\
\hline Idade & \\
De 18 a 29 anos & $29,28 \%$ \\
De 30 a 39 anos & $27,85 \%$ \\
De 40 a 49 anos & $22,85 \%$ \\
De 50 a 59 anos & $18,57 \%$ \\
De 60 anos ou mais & $01,42 \%$ \\
\hline
\end{tabular}

Autor: SCHONS, MAY, MULLER, ALBUQUERQUE, 2021.

Tabela 2 - Perfil profissional dos profissionais de saúde.

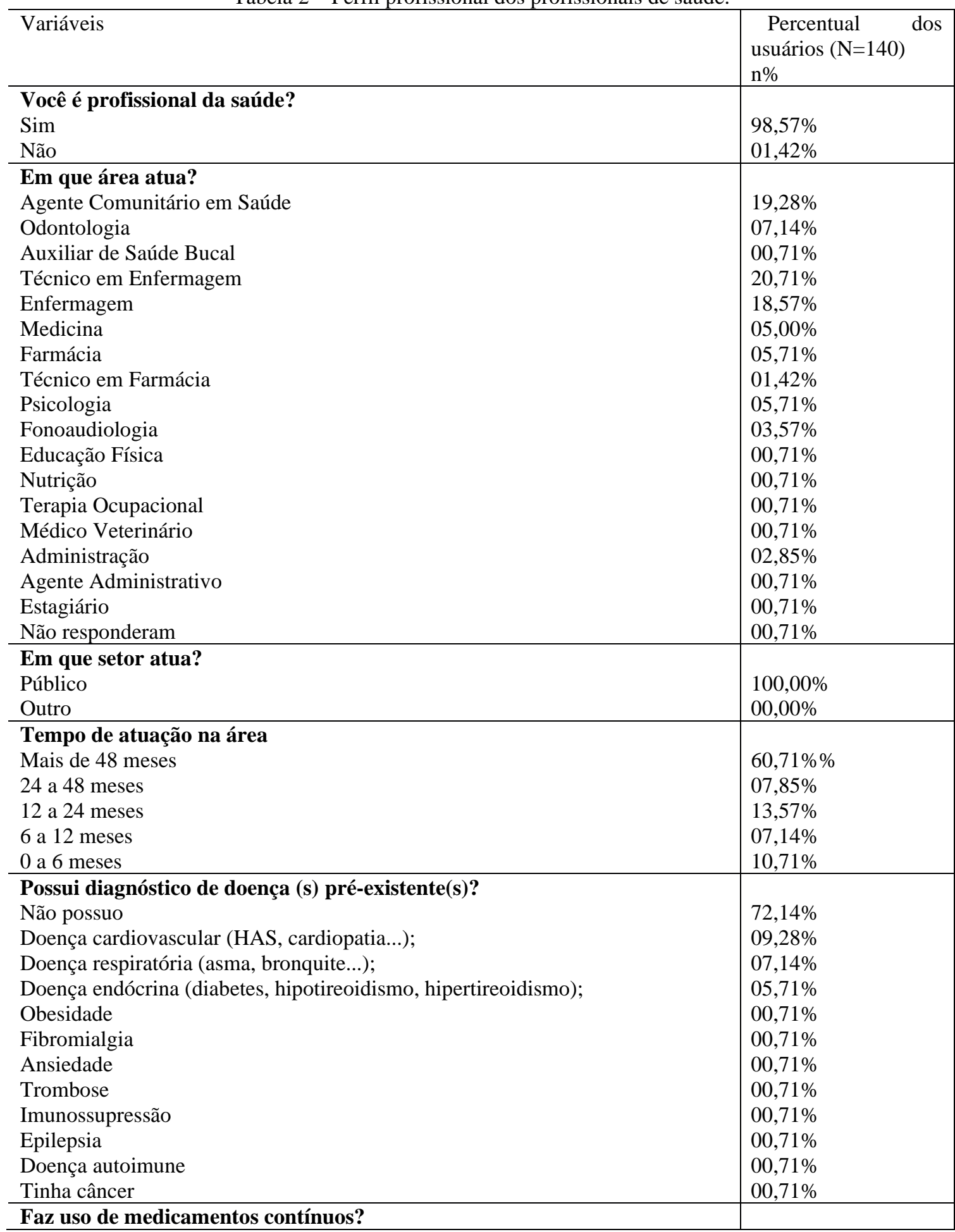




\begin{tabular}{l|l|}
\hline Sim & $40,00 \%$ \\
Não & $60,00 \%$ \\
\hline Senti medo relacionado à Pandemia de COVID 19? & \\
Razoavelmente & $50,00 \%$ \\
Muito & $32,14 \%$ \\
Pouco ou nenhuma & $17,85 \%$ \\
\hline Com a chegada da COVID 19, tem feito uso de alguma substância lícita ou \\
ilícita (álcool, tabaco, maconha, cocaína...). & \\
Sim & $21,42 \%$ \\
Não & $78,57 \%$ \\
\hline
\end{tabular}

Autor: SCHONS, MAY, MÜLLER, ALBUQUERQUE, 2021.

Tabela 3 - Sentimentos vivenciados pelos profissionais de saúde frente à pandemia.

\begin{tabular}{|c|c|}
\hline $\begin{array}{l}\text { Tive alterações no SONO (insônia, sonhos perturbadores, dificuldade para } \\
\text { adormecer...). } \\
\text { Nenhum dia } \\
\text { Em } 1 \text { a } 2 \text { dias na semana } \\
\text { Em } 3 \text { a } 4 \text { dias na semana } \\
\text { Em } 5 \text { a } 6 \text { dias na semana } \\
\text { Todos os dias }\end{array}$ & $\begin{array}{l}36,42 \% \\
34,28 \% \\
18,57 \% \\
02,85 \% \\
05,00 \%\end{array}$ \\
\hline $\begin{array}{l}\text { Senti-me ansioso (a), agitado (a), inquieto (a) desde o inicio da pandemia: } \\
0 \\
1 \\
2 \\
3 \\
4 \\
5\end{array}$ & $\begin{array}{l}05,71 \% \\
11,42 \% \\
20,00 \% \\
24,28 \% \\
19,28 \% \\
19,28 \%\end{array}$ \\
\hline $\begin{array}{l}\text { Senti-me TRISTE } \\
0 \\
1 \\
2 \\
3 \\
4 \\
5\end{array}$ & $\begin{array}{l}12,14 \% \\
12,85 \% \\
23,57 \% \\
17,85 \% \\
17,85 \% \\
15,71 \%\end{array}$ \\
\hline $\begin{array}{l}\text { Senti-me MOTIVADO (A) / ENTUSIASMADO (A) } \\
0 \\
1 \\
2 \\
3 \\
4 \\
5\end{array}$ & $\begin{array}{l}17,14 \% \\
23,57 \% \\
22,14 \% \\
20,71 \% \\
09,28 \% \\
07,14 \%\end{array}$ \\
\hline $\begin{array}{l}\text { Senti meu coração acelerado mesmo não tendo feito esforço físico } \\
0 \\
1 \\
2 \\
3 \\
4 \\
5\end{array}$ & $\begin{array}{l}26,42 \% \\
12,85 \% \\
12,14 \% \\
19,28 \% \\
17,85 \% \\
11,42 \%\end{array}$ \\
\hline $\begin{array}{l}\text { Tive suores, mal-estar? } \\
0 \\
1 \\
2 \\
3 \\
4 \\
5\end{array}$ & $\begin{array}{c}40,00 \% \\
16,42 \% \\
10,00 \% \\
17,14 \% \\
09,28 \% \\
07,14 \% \\
\end{array}$ \\
\hline $\begin{array}{l}\text { Tenho sido capaz de desfrutar de atividades de lazer } \\
\text { Não tenho conseguido }\end{array}$ & $32,85 \%$ \\
\hline
\end{tabular}




\begin{tabular}{l|l|}
\hline Eventualmente & $57,14 \%$ \\
Todos os dias & $05,00 \%$ \\
Não tenho / tinha atividades & $05,00 \%$ \\
\hline
\end{tabular}

Autor: SCHONS, MAY, MULLER, ALBUQUERQUE, 2021.

Tabela 4 - Segurança e capacitação no trabalho durante a pandemia da COVID-19

\begin{tabular}{l|l|}
\hline Tenho a possibilidade de falar com alguém sobre meus & \\
problemas no trabalho ou em casa & $08,57 \%$ \\
0 & $08,57 \%$ \\
1 & $15,71 \%$ \\
2 & $16,42 \%$ \\
3 & $23,57 \%$ \\
4 & $17,14 \%$ \\
5 & \\
\hline Sente-se seguro (a) em seu local de trabalho? & $45,00 \%$ \\
Sim & $55,00 \%$ \\
Não & \\
\hline Você se afastaria do seu local de trabalho nesse período? Se sim, \\
por qual dos motivos: & $12,14 \%$ \\
Me afastaria por medo de me contaminar & $51,42 \%$ \\
Não me afastaria & $00,71 \%$ \\
Doença autoimune & $01,42 \%$ \\
Me afastaria por me sentir inseguro no local de trabalho (falta de & \\
EPI’S) & $25,71 \%$ \\
Me afastaria para proteger os meus familiares & $04,28 \%$ \\
Já estou afastado por motivos de saúde & $00,71 \%$ \\
Se necessário independente do caso & $00,71 \%$ \\
Se tiver doente com sintomas & $01,42 \%$ \\
Férias & \\
\hline Você se sente sobrecarregado (a)? & \\
Sim & \\
Não & $28,57 \%$ \\
Consideravelmente & $22,85 \%$ \\
\hline O quanto você se sente capacitado (a) tecnicamente para atuar & \\
no enfrentamento e controle da COVID-19? & \\
\hline & \\
\hline & \\
\hline
\end{tabular}




\begin{tabular}{l|l|}
\hline Pouco capacitado (a) & $25,00 \%$ \\
Mais ou menos capacitado (a) & $57,85 \%$ \\
Nada capacitado (a) & $08,57 \%$ \\
Muito capacitado (a) & $08,57 \%$ \\
\hline
\end{tabular}

Autor: SCHONS, MAY, MULLER, ALBUQUERQUE, 2021.

\section{DISCUSSÃO}

O município de Santa Rosa está localizado no noroeste do estado do Rio Grande do Sul, com uma área territorial de 488,4 quilômetros quadrados, com economia predominante metal mecânico e agrícola, sendo também conhecida como Berço Nacional da Soja. A região é modelo do segmento industrial no setor polo metal mecânico, fabricando peças, máquinas e implementos agrícolas, movimentando o agronegócio e produzindo 66\% das colheitadeiras brasileiras (PMSR, 2016).

A necessidade de inovação para melhor eficácia na gestão municipal da saúde com base na busca da universalidade do acesso, ações e serviços e na democratização das relações com a sociedade, impulsionou a implantação do Sistema Único de Saúde no município de Santa Rosa. Houve priorização da política de saúde aos princípios do SUS em 1993, passando a ser um dos dois primeiros municípios no Estado do Rio Grande do Sul a efetivar a municipalização (GALLO, 2007).

Em meados da década de 90 emerge, no cenário brasileiro, a proposição de reorganização do modelo assistencial de saúde e a partir desta proposta criada, em 1994, o Programa de Saúde da Família (PSF) que, na década seguinte, reorganiza-se em Estratégia de Saúde da Família (ESF). A ESF é a porta de entrada para a população no Sistema Único de Saúde (SUS), representando um mecanismo emblemático de reorganização da Atenção Primária em Saúde (APS) em nosso país (BRASIL, 2012). Em sua forma mais desenvolvida, a APS é o primeiro contato com o sistema de saúde e o local responsável pela organização do cuidado à saúde dos indivíduos, suas famílias e da população ao longo do tempo (STARFIELD, 2002).

A partir das considerações feitas acima e com o princípio da descentralização a Fundação Municipal de Saúde assume a total responsabilidade pelas ações e estratégias de ampliação da política pública de saúde em seu território tendo como foco principal a APS. A APS possui diversos mecanismos e capilaridades a fim de atender as demandas peculiares dos sujeitos e do local em que vivem, resultando nas diferentes ofertas e ações de serviços. Para isso o Ministério da Saúde propõe diversas áreas de atuações a fim de atender esses cenários plurais, tais como: serviços na área de saúde mental como Centros de Atenção Psicossocial (CAPS), equipes de apoio nos territórios dos ESF Núcleo de Apoio à Saúde da Família (NASF), academias populares de saúde, serviços especializados entre outros. 
O Centro de Referência em Saúde do Trabalhador - CEREST está sediado no município de Santa Rosa - RS, tendo sua abrangência na região Fronteira Noroeste, contemplando um total de 22 (vinte e dois) municípios da $14^{\circ}$ Região de Saúde do Rio Grande do Sul.

Conforme Portaria do Ministério da Saúde no 2.728/2009 que dispõe sobre a Rede Nacional de Atenção Integral à Saúde do Trabalhador (RENAST) e dá outras providências, os Centros de Referência em Saúde do Trabalhador - CEREST tem por função dar subsídio técnico para o SUS, nas ações de promoção, prevenção, vigilância, diagnóstico, tratamento e reabilitação em saúde dos trabalhadores urbanos e rurais.

No contexto do SUS a área de Saúde do Trabalhador emerge como um desafio a mais, no sentido de promover os meios necessários para atendimento com primazia, o que a partir de 1988 com a Constituição Federal, passou a ser também, atribuição das Secretarias de Saúde dos Estados e Municípios.

O CEREST - Fronteira Noroeste em Santa Rosa é composto pelos seguintes profissionais na área técnica: Enfermeira, Fisioterapeuta, Fonoaudióloga, Técnica em Enfermagem, Técnico em Segurança do Trabalho, Médica e Psicóloga.

É neste contexto que esta pesquisa está inserida, tendo na linha de frente no combate ao COVID19, os profissionais técnicos em enfermagem (20,71\%), enfermeiros (18,57\%), agentes comunitários de saúde $(19,28 \%)$ odontólogos $(07,14 \%)$, psicólogos $(05,71 \%)$ médicos $(05,00 \%)$ e demais capacitados, realizam os atendimentos a população nos estabelecimentos de saúde e nos domicílios, adotando os protocolos de controle de infecção (contato, via aérea) EPIs, incluindo máscaras N95, aventais, óculos, protetores faciais e luvas permitindo a segurança do profissional e do paciente. Sendo que $100 \%$ dos entrevistados são profissionais de saúde que atuam no Sistema Único de Saúde (SUS) e destes, 60\% têm mais de dois anos de atuação, no momento da pesquisa, no SUS.

No entanto, conforme Duarte, Silva e Bagatini (2021), sabe-se que, diante do cenário atual, há escassez de EPIs em vista disso orienta-se o uso de máscaras faciais de tecido para a população em geral, já que as descartáveis precisam ser destinadas a estes profissionais. Além disso, a falta desses equipamentos também é fonte geradora de medo e angústia para equipe.

A exposição dos profissionais de saúde no cuidado direto a paciente com o vírus tem influência na sua saúde mental, impactando nos processos de trabalho e na vida pessoal desses trabalhadores (DUARTE, SILVA e GABATINI, 2021). Nesse sentido, essa pesquisa mostra um número expressivo desses profissionais que relataram apresentar sintomas relacionados a alterações com o sono (insônia, sonhos perturbadores, dificuldade para adormecer...) responderam; nenhum dia (36,42\%), em um a dois dias na semana $(34,28 \%)$ em três a quatro dias na semana $(18,57 \%)$ e todos os dias $05,00 \%$, ao mesmo tempo esses profissionais relataram sentirem-se ansioso/agitado (19,28\%); triste (23,57\%). Esses sintomas provavelmente possuem relação com o estigma de os profissionais por terem contato direto com os 
pacientes contaminados, assim como maior demanda no ambiente de trabalho, e a necessidade de atualização constante $(\mathrm{CH}, 2020)$.

Já quando questionados sobre, o sentimento de medo relacionado à pandemia, 50\% responderam razoável, muito 32,14\% e 17,85\% relataram pouco ou nenhum. Esses mistos de sentimentos são uma reação natural diante de uma ameaça real e proeminente, que demanda muitas vezes um agir racional para seu enfrentamento, pautado em informações realistas e concretas, com a finalidade de contribuir, assim para as medidas de proteção acessíveis (JODELET, 2019).

Por conta disso, o cenário desolador pode provocar quadros de burnout (estresse relacionado ao trabalho), que é compreendido por Silva et. al (2015), como um esgotamento profissional causado por uma síndrome psicológica decorrente da tensão emocional crônica vivida pelo trabalhador, associada a despersonalização e baixa realização pessoal. Tendo em vista que 48,57\% dos entrevistados sentem-se sobrecarregados profissionalmente, e não se sentem seguros no campo de trabalho $(55,00 \%)$ em função do aumento de risco no adoecimento ou por contaminação, oferecendo insegurança para realizar procedimentos, mas ao mesmo tempo não deixariam de realizar a assistência à população (51,42\%). Este estado de tensão emocional e estresse crônico, provocado por condições de trabalho físicas, emocionais e psicológicas desgastantes faz com que, pessoas cuja profissão exige envolvimento interpessoal direto e intenso, adoeçam. Profissionais das áreas de educação, saúde, assistência social, recursos humanos, agentes penitenciários, bombeiros, policiais e mulheres que enfrentam dupla jornada correm risco maior de desenvolver a síndrome de burnout.

Burnout na saúde é um fenômeno complexo e multidimensional resultante da interação entre aspectos individuais e o ambiente de trabalho, a ocorrência em profissionais da saúde tem sido considerada um fenômeno psicossocial relevante, pois afeta não somente o profissional, mas também o ambiente de trabalho, interferindo na obtenção dos objetivos terapêuticos, uma vez que os profissionais acometidos pela síndrome desenvolvem um processo de alienação, desumanização e apatia. Burnout é o resultado do estresse crônico, típico do cotidiano do trabalho, principalmente quando neste existem excessiva pressão, conflitos, poucas recompensas emocionais e pouco reconhecimento, sendo considerado um fenômeno psicossocial constituído de três dimensões: Exaustão Emocional, Despersonalização e Baixa Realização Profissional. O "Esgotamento Emocional” caracteriza-se pela sensação de exaustão emocional e física, na qual o sujeito constata que não possui energias suficientes para continuar a trabalhar; A "Despersonalização" representa a dimensão do contexto interpessoal do burnout e refere-se a atitudes de distanciamento emocional direcionadas às pessoas a quem o sujeito deve prestar serviços, bem como aos colegas de trabalho; E a "Falta de Realização" corresponde à dimensão da autoavaliação do burnout e, tal como o nome indica, refere-se a sentimentos de incompetência e de baixa produtividade no trabalho, bem como de descontentamento a nível pessoal. 
O sintoma típico da síndrome de burnout é a sensação de esgotamento físico e emocional que se reflete em atitudes negativas, como ausências no trabalho, agressividade, isolamento, mudanças bruscas de humor, irritabilidade, dificuldade de concentração, lapsos de memória, ansiedade, depressão, pessimismo, baixa autoestima. Além disso, pode haver a presença de dor de cabeça, enxaqueca, cansaço, sudorese, palpitação, pressão alta, dores musculares, insônia, crises de asma, distúrbios gastrintestinais são manifestações físicas que podem estar associadas à síndrome, sinais e sintomas estes referidos pelos profissionais de saúde na pesquisa realizada. O tratamento da Síndrome de Bournout inclui o uso de antidepressivos e psicoterapia. Atividade física regular e exercícios de relaxamento também ajudam a controlar os sintomas, melhorando a qualidade de vida.

É necessário avaliar se é o ambiente profissional que causa o estresse ou se são as atitudes da própria pessoa que passam a ser o estopim. Existem três focos durante o tratamento psicoterápico: a relação com a profissão, o ambiente de trabalho e o trabalho com foco nos sintomas - por exemplo, a dificuldade de concentração. Junto à terapia, os especialistas aconselham melhorar a qualidade de vida, prevenir o estresse, garantir boa saúde física, dormir e alimentar-se bem, praticar atividades físicas e manter hobbies e interesse pela vida social. Especialistas orientam não usar a falta de tempo como desculpa para não praticar exercícios físicos e não desfrutar momentos de descontração e lazer. Quanto à realização de atividades de lazer durante a pandemia, os profissionais de saúde entrevistados referiram realizar eventualmente $(57,14 \%)$ e não realizar (32,85\%). Desta forma, é urgente mudanças no estilo de vida para prevenir ou tratar a síndrome de burnout, além de avaliar a possibilidade de propor novas dinâmicas para as atividades diárias e objetivos profissionais, devendo ter este foco os serviços de saúde que pretendem realizar o enfrentamento a síndrome de burnout em seus trabalhadores.

Em relação à segurança e saúde dos profissionais de saúde, amplamente discutido em diversas publicações, ressalta-se que uma força de trabalho capacitada e motivada esta diretamente relacionada aos investimentos realizados na promoção da saúde e segurança do trabalhador (SILVA-JUNIOR, 2017). Neste sentido, as respostas dos trabalhadores de saúde questionados quanto se sentirem seguros no local de trabalho, refletem na boa gestão e condução de recursos financeiros e pessoais investidos no município.

Posto isto, consideramos a relação de segurança dos trabalhadores com seu ambiente de trabalho pode estar relacionada à estabilidade no serviço de saúde, ao plano de carreiras dos servidores municipais e vínculos formais de trabalho.

Salientando a realidade local, vários projetos e ações de saúde ocupacional surgem a fim de minimizar as mudanças na rotina de trabalho durante a pandemia e contribuir para a manutenção da saúde do profissional que está na linha de frente. Destacamos as ações desenvolvidas pela instituição FUMSSAR, juntamente do CEREST Fronteira Noroeste e o Núcleo de Ensino e Pesquisa (NEP), no âmbito de educação 
em saúde e condições de trabalho visam reduzir os impactos na rede de assistência e na saúde de seus trabalhadores, preparando o sistema e servidores frente à nova situação.

Barroso et al. (2020) ressaltam a necessidade de ações e reflexões acerca dos processos de trabalho, interlocução entre gestores e servidores, capacitações e treinamentos constantes dos trabalhadores.

Conforme Almeida (2020), os desafios emergentes a nova situação manifestada em várias localidades podem estar relacionados ao escasso tempo de preparo e a necessidade de implementação imediata de ações efetivas no controle da COVID-19, associada as dificuldades e carências que o serviço de saúde já apresentava.

Frente essa necessidade de reestruturação e resposta rápida e eficaz para controle da pandemia, destacamos a criação do Comitê Intersetorial de Enfrentamento a COVID-19, a organização de unidades sentinelas em 3 regiões do município para atendimento de indivíduos com sintomas respiratórios, e pactuada rede intersetorial com vistas a garantir e organizar o fluxo dos pacientes com síndrome respiratória, contando com a implantação do serviço hospitalar de unidade Especializada de tratamento a COVID-19 e o serviço da Unidade de Pronto Atendimento (UPA), ambos como referência de internação, alguns deles a nível regional.

Enfatizando os dados obtidos no que se refere à sensação de segurança no local de trabalho pelos profissionais de saúde, o sistema se sobressai nas ações realizadas no enfrentamento a COVID-19 ao dispor de uma rede de serviços locais, disponibilidade de equipamentos e recursos humanos. Tais medidas, adotadas também por outros estados brasileiros, têm reconhecido o trabalhador do SUS como linha de frente imprescindível no combate à pandemia.

Corroborando com o que vem sendo realizado no âmbito local, Barroso et al. (2020), substância a necessidade de medidas de proteção, capacitação e oferta de condições de trabalho adequadas para os profissionais dos estabelecimentos de saúde, sobretudo, maior destinação de recursos a tais medidas, contratação de maior número de profissionais.

Em diferentes localidades do país e do mundo, muitos profissionais da área da saúde estão atuando diretamente na linha de frente desde o início da pandemia. Com isso, além da disseminação sem precedentes do vírus e a capacidade do sistema de saúde em dar conta de tal situação, em muito se faz necessário a real avaliação de como esses profissionais se encontram no exercício de suas respectivas funções. A ansiedade nos profissionais de saúde durante a pandemia pela Covid-19

As condições de trabalho de diferentes profissionais dentre médicos, enfermeiros, técnicos em enfermagem entre outros, a médio e longo prazo se torna um desafio, principalmente em relação há quanto tempo permanecerão ativos para o bom desempenho de suas atividades. Além disso, faz repensar que além das aflições percebidas pela maioria das pessoas, estes "heróis sem capa" sofrem por turnos de trabalho extensos, maior risco de exposição ao vírus, mudanças em seus respectivos ambientes laborais, além das 
questões morais que envolvem diretamente seus familiares e a insegurança caso se contaminem e por consequência acabam por contaminar algum ente querido. A ansiedade nos profissionais de saúde durante a pandemia pela Covid-19

Estes diferentes cenários vivenciados diariamente pelos profissionais de saúde atuantes da linha de frente: efetivos de trabalhadores reduzidos por comporem grupos de risco, sobrecarga de trabalho aos demais atuantes, jornadas prolongadas de trabalho, além dos citados anteriormente, apenas reforçam o quanto este cenário degradante aumenta as chances de sofrimento mental, sobrecarga física, gerando frustrações e ansiedades sem precedentes para esses trabalhadores (ALMEIDA, 2020).

Quando relatado a saúde mental, além das questões vividas diretamente pelos profissionais, o método repercutido de distanciamento social, também impacta diretamente a população, principalmente no que diz respeito às relações interpessoais totalmente modificadas e muitas vezes prejudicadas nesse período de pandemia.

Neste cenário preocupante em relação à saúde mental dos trabalhadores o que se salienta é a importância do Estado em voltar às atenções para esta realidade, sendo que muitos dos danos ocasionados pelo período pandêmico serão notados após o seu término. Para isso, a dedicação de esforços individuais e coletivos nas diferentes áreas do conhecimento, poderão reduzir os impactos negativos na saúde mental desses profissionais.

Ademais, os profissionais da saúde quando questionados sobre o "quanto se sente capacitado (a) tecnicamente para atuar no enfrentamento e controle da COVID-19”, 25\% responderam que se sentem pouco capacitado (a), 57,85\% mais ou menos capacitados (as), 08,57\% nada capacitado (a) e 08,57\% sentem-se muito capacitados (as). O Ministério da Saúde lançou no início de abril de 2020 a ação estratégica "Brasil conta comigo - Profissionais da Saúde", que teve como objetivo o cadastro e capacitação de profissionais que estivessem dispostos a atuar no combate ao COVID-19 no país, ficando à disposição de gestores federais, estaduais, distritais e municipais do SUS (SOUZA E SOUZA e SOUZA, 2020).

\section{CONCLUSÕES OU CONSIDERAÇÕES FINAIS}

Os resultados obtidos neste trabalho auxiliam no delineamento da dimensão ocupacional em profissionais da saúde durante a pandemia. Fatores como os sentimentos de medo quando da possibilidade de contaminação de familiares, transparece os elementos que constituem o sujeito para além do trabalho exercido, humanizando tais cuidadores. Cabe aqui que ressaltar que as dimensões de planejamento e de gestão em saúde considerem as peculiaridades em que os trabalhadores se encontram neste momento.

Os achados até aqui também reforçam o que a literatura científica, normas e legislações da saúde do trabalhador afirmam da importância de proporcionar um ambiente de trabalho seguro faz-se necessário. Em especial neste período de pandemia, as garantias de um ambiente seguro para profissionais de saúde. 
Compreende-se dessa maneira, que a pandemia o qual estamos vivenciando é multidimensional, com impactos em todas as dimensões, incluindo físico, emocional, econômico, social e psicológico. Sendo assim, embora o medo e a ansiedade sejam esperados durante esse período, também é importante entendermos e identificarmos de maneira precoce para darmos respostas adequadas a tais sinais e sintomas.

Pensando nisso, acreditamos que a prevenção, promoção em saúde e ações de educação em saúde são ideias nesse momento, com a presença e o amparo de psicólogos, psicoterapeutas e psiquiatras nos centros hospitalares e nas Unidades de atendimentos com a finalidade de prestarem uma assistência/suporte aos profissionais. Desse modo, em síntese é fundamental que os especialistas precisam dispor de formações específicas para lidar com o tipo de demanda, sabendo manejar os casos corretamente e lidando com as necessidades de saúde mental dos profissionais de saúde.

Consideramos importante, o reconhecimento e a valorização/enaltecimento aos profissionais envolvidos no combate a pandemia da Covid-19, visando a sua importância nesse momento tão delicado que a sociedade vive, pois muitos estão realizando horas extras de trabalho, consumindo energias acima do normal e ao mesmo tempo acabam deixando de gozar das atividades em lazer com suas famílias com o propósito de não os expor.

Diante o cenário pandêmico o qual enfrentamos, é possível salientar e manifestar o quão importante é o apoio dos cidadãos aos profissionais de saúde que estão na linha de frente prestando a assistência de maneira direta e indireta à população em condições que nem sempre são seguras. Isso tudo, contribui para o reconhecimento do protagonismo dos profissionais de saúde pública como ordenadora do cuidado no SUS.

Espera-se que esta pesquisa auxilie na tomada de decisões em saúde do trabalhador de saúde, visando melhorias na qualidade e segurança do trabalho, que proporcionará qualidade de vida e promoção da saúde aos profissionais que atuam na linha de frente a pandemia da COVID-19. 


\section{REFERÊNCIAS}

ALMEIDA, I.M. de. Proteção da saúde dos trabalhadores da saúde em tempos de COVID-19 e respostas à pandemia. Rev. bras. saúde ocup., São Paulo, v.45, n.17, 2020.

BARROSO, B.I.L.; SOUZA, M.B.C.A.; BREGALDA, M.M.; LANCMAN, S.; COSTA, V.B.B. Saúde do trabalhador em tempos de covid-19: reflexões sobre saúde, segurança e terapia ocupacional. Rev. bras. saúde ocup., São Paulo, no prelo, 2020.

BRASIL. Ministério da Saúde. Política Nacional de Atenção Básica (PNAB). Brasília: Ministério da Saúde, 2012.

DUARTE, Maria de Lourdes Custódio; SILVA, Daniela Giotti da; BAGATINI, Mariana Mattia Correa. Enfermagem e saúde mental: uma reflexão em meio à pandemia de coronavírus. Rev. Gaúcha Enferm. Vol.42, Porto Alegre, 2021.

FUNDAÇÃO MUNICIPAL DE SAÚDE DE SANTA ROSA - FUMSSAR. Plano Municipal de Saúde do Município de Santa Rosa 2016-2019. Santa Rosa: FUMSSAR, 2016.

GALLO, Z.M.C. A ética discursiva dos atores da política de saúde de Santa Rosa. 2007, 143f. Tese (Doutorado em Serviço Social) - Pontifícia Universidade Católica do Rio Grande do Sul, Porto Alegre, 2007.

IBGE - INSTITUTO BRASILEIRO DE GEOGRAFIA E ESTATISTICA. Rio de Janeiro: 2020. Disponível em: $\quad$ http://cidades.ibge.gov.br/xtras/perfil.php?lang=\&codmun=431720\&search=\|infogr\%E1ficos:informa\%E7\%F5es-completas Acesso em: maio.2020.

CH - INTER-AGENCY STANDING COMMITTEE IASC's Reference Group on Mental Health and Psychosocial Support. Interim briefing note addressing mental health and psychosocial aspects of COVID19 outbreak. Geneva: IASC; 2020 [cited 2020 Apr 15]. Available from: https://interagencystandingcommittee.org/iasc-reference-group-mental-health-and-psychosocial-supportemergency-settings/interim-briefing-note-addressing-mental-health-and-psychosocial-aspects-covid-19outbreak

JODELET, Denise. Abordagem psicossociológica sobre ameaças e seus usos sociais, [s. l.], 2019. Disponível em: https://www.scielo.br/j/se/a/9RKx5XjMDb98qHWfDrnDVxQ/?lang=pt

PREFEITURA MUNICIPAL DE SANTA ROSA- PMSR. Santa Rosa: 2016. Disponível em: http://www.santarosa.rs.gov.br/index2.php Acesso em: maio. 2020.

RAVAGNi E. O que é Sexualidade Humana? 2007. Tese de Doutorado pela Universidade de Brasília, Faculdade de Ciências da Saúde. Brasília: 2007. Disponível em: https://repositorio.unb.br/handle/10482/1226 Acesso em: mar 2021

SAMPIERI, R.H.; COLLADO, L.C.F., BAPTISTA, M. D.P. Metodologia de Pesquisa. $5^{\text {a }}$ ed. Porto Alegre: Penso, 2013.

SILVA, Jorge Luiz Lima da et al. Fatores psicossociais e prevalência da síndrome de burnout entre trabalhadores de enfermagem intensivistas. Rev. bras. ter. intensiva. 2015, vol.27, n.2 pp.125-133. 
SILVA-JUNIOR, J.S. A saúde do trabalhador que cuida da saúde. Rev Bras Med Trab., v.15, n.3, 2017. SOUZA E SOUZA LPS, SOUZA AG. Enfermagem brasileira na linha de frente contra o novo Coronavírus: quem cuidará de quem cuida? Journal of nursing and health. 2020.

STARFIELD, B. Atenção primária: equilíbrio entre necessidades de saúde, serviços e tecnologia. Brasília, UNESCO/Ministério da Saúde, 2002. 